\title{
Effect of functional monomers in all-in-one adhesive systems on formation of enamel/dentin acid-base resistant zone
}

\author{
Toru NIKAIDO ${ }^{1}$, Chiaki ICHIKAWA ${ }^{1}, \mathrm{Na} \mathrm{LI}^{2}$, Tomohiro TAKAGAKI ${ }^{1}$, Alireza SADR ${ }^{3}$, Yasuhiro YOSHIDA ${ }^{4,5}$, \\ Kazuomi SUZUKKI ${ }^{4,5}$ and Junji TAGAMI ${ }^{1,3}$
}

\author{
${ }^{1}$ Cariology and Operative Dentistry, Department of Restorative Sciences, Graduate School of Medical and Dental Sciences, Tokyo Medical and \\ Dental University ,1-5-45, Yushima, Bunkyo-ku, Tokyo 113-8549, Japan \\ ${ }^{2}$ Department of Prosthodontics, School of Stomatology, Fourth Military Medical University, No.17, Changle West Road, Xi'an, Shaanxi, 710032,P.R., \\ China \\ ${ }^{3}$ Global Center of Excellence (GCOE) Program; International Research Center for Molecular Science in Tooth and Bone Diseases, Tokyo Medical \\ and Dental University, 1-5-45, Yushima, Bunkyo-ku, Tokyo 113-8549, Japan \\ ${ }^{4}$ Department of Biomaterials, Okayama University Graduate School of Medicine, Dentistry and Pharmaceutical Sciences, 2-5-1 Shikata-cho, \\ Okayama 700-8558, Japan \\ ${ }^{5}$ Research Center for Biomedical Engineering, Okayama University, 2-5-1 Shikata-cho, Okayama 700-8558, Japan \\ Corresponding author, Toru NIKAIDO; E-mail: nikaido.ope@tmd.ac.jp
}

\begin{abstract}
This study aimed at evaluating the effect of functional monomers in all-in-one adhesive systems on formation of acid-base resistant zone (ABRZ) in enamel and dentin. Experimental adhesive systems containing one of three functional monomers; MDP, 3D-SR and 4-META were applied to enamel or dentin surface and light-cured. A universal resin composite was then placed. The specimens were subjected to a demineralizing solution $(\mathrm{pH} 4.5)$ and $5 \% \mathrm{NaClO}$ for acid-base challenge and then observed by SEM. The ABRZ was clearly observed in both enamel and dentin interfaces. However, enamel ABRZ was thinner than dentin ABRZ in all adhesives. Morphology of the ABRZ was different between enamel and dentin, and also among the adhesives. Funnel-shaped erosion was observed only in the enamel specimen with the 4-META adhesive. The formation of enamel/dentin ABRZ was confirmed in all adhesives, but the morphology was influenced by the functional monomers.
\end{abstract}

Keywords: Acid-base resistant zone, All-in-one adhesive system, Functional monomer, Hybrid layer chemical bonding

\section{INTRODUCTION}

Since the introduction of adhesion to dentistry, the dental adhesives have been remarkably simplified and improved. In the recent years, all-in-one adhesive systems, which combine etching, priming and bonding into a single application step have been widely accepted by the dentists for bonding of direct composite restorations.

Most of the all-in-one adhesive systems contain one or more functional monomers, which have important roles such as etching the tooth substrates, enhancing monomer penetration, and also imparting the adhesives with chemical interaction potential ${ }^{1,2)}$. Yoshida et al. ${ }^{3)}$ reported that the chemical bonding potential was different among various functional monomers. Comparing three commonly used functional monomers, they observed that chemical bonding potential was the highest in 10-methacryloxydecyl dihydrogen phosphate (MDP), followed by 4-methacryloxyethyl trimellitate anhydride (4-META) and 2-methacryloyloxyethyl phenyl phosphate (Phenyl-P). The capability of MDP to readily establish an intensive ionic bond with hydroxyapatite (HAp) has been demonstrated.

Such a chemical interaction with between the functional monomer of an adhesive system and HAp may result in modification of the bonding substrate in favor of longevity of the dental restoration ${ }^{4}$. In this regard,
Tsuchiya et al.5) reported the formation of a new zone beneath the hybrid layer when dentin was treated with a self-etching adhesive system. The zone was different from the conventional hybrid layer ${ }^{6}$, and characterized by resistance to an acid-base challenge. Therefore, this zone named as the "acid-base resistant zone" (ABRZ), was supposed to play an important role in prevention of secondary caries, sealing of restoration margins and promotion of restoration durability. In previous studies, the existence of the dentin ABRZ was confirmed with various self-etching adhesive systems ${ }^{7-13)}$. Furthermore, the transmission electron microscopic (TEM) observation of the adhesive-dentin interface after acid-base challenge revealed that the ABRZ contained HAp crystals and possessed a dentin-like structure, more caries-resistant than normal dentin ${ }^{9}$. The attributes of ABRZ could suggest that the bonding technology could reinforce dentin against acid-attack, and it was proposed that such a reinforced dentin could be called as "super dentin" ${ }^{10,14)}$.

While earlier studies focused on dentin ABRZ, some more recent works have focused on enamel as well. Li et $a l .{ }^{15)}$ investigated the effects of two functional monomers of MDP and Phenyl-P contained in two-step self-etching adhesive systems on the morphology of adhesive-enamel interface after acid-base challenge. They found that formation of enamel ABRZ was confirmed along the adhesive-enamel interface only when the experimental 
adhesives contained MDP, as an enamel ABRZ was not observed with the adhesive system which contained Phenyl-P in both the primer and the bonding agents. From the previous study, it was supported that the formation of the enamel ABRZ was influenced by selection of the functional monomers.

Most of the ABRZ studies to date have used commercially marketed adhesives with various compositions, making it difficult to highlight the role of a specific component. Moreover, there is little information about the ABRZ formation on enamel and dentin in all-in-one adhesive systems. MDP, the three dimensional self-reinforcing monomer (3D-SR) and 4-META are three functional monomers used in the commercially available all-in-one adhesive systems now. Therefore, the purpose of this study was to evaluate the effect of experimental all-in-one adhesives containing one of three different functional monomers; MDP, 3D-SR and 4-META on morphological characteristics of the enamel/dentin ABRZ. The hypothesis of this study was that the enamel/ dentin ABRZ formation was influenced by the type of the functional monomers in the all-in-one adhesive systems.

\section{MATERIALS AND METHODS}

\section{Materials used in this study}

The compositions of the experimental all-in-one adhesives are listed in Table 1 . The experimental all-in-one adhesive systems were basically composed of one of the functional monomers; MDP, 3D-SR and 4-META, base monomers, solvents (water and acetone) and photo-initiators all mixed in to one bottle. The compositions were similar in all aspects except for the functional monomer. The chemical formulae of the three functional monomers were illustrated in Fig. 1. These functional monomers used in this study were prepared according to the methodologies in the previous studies ${ }^{16-18)}$. The name codes for the three experimental all-in-one adhesives were MDP, 3D-SR and 4-META, respectively. The $\mathrm{pH}$ value of adhesive was 1.9 in MDP, 2.0 in 3D-SR, and 2.2 in 4-META, measured using a portable $\mathrm{pH}$ meter (B-213 Twin Compact Meter, Horiba, Kyoto, Japan). The acidic $\mathrm{pH}$ value of 4-META adhesive was due to formation of 4-methacryloxyethyl trimellitate (4-MET) after hydrolysis of 4-META with water in the adhesive.

Directions for use of the experimental all-in-one adhesive systems are as follows; the adhesive was applied to enamel/dentin for $20 \mathrm{~s}$, mildly air dried and then strongly air dried for $5 \mathrm{~s}$ each, and light cured for 10 s using a halogen light curing unit (Optilux 500, Demetron-Kerr, Danbury, CT, USA).

Fifteen non-carious human third molars and fifteen human premolars were collected after the individuals' informed consent was obtained according to a protocol approved by the Institutional Review Board of Tokyo Medical and Dental University. The teeth were stored in water at $4^{\circ} \mathrm{C}$ and used within 1 month after extraction.

\section{Specimen preparation for dentin $A B R Z$}

The sample preparations for SEM examination of the enamel/dentin ABRZ were illustrated in Fig. 2. The procedures previously reported by Inoue et al. ${ }^{7}$ ) became the standard for ABRZ observation studies carried out later on. For the dentin specimens, human molars were sectioned at the mid coronal portion, vertical to the tooth axis, with a low-speed diamond saw (Isomet, Buehler, Lake Bluff, IL, USA) to obtain $1 \mathrm{~mm}$-thick dentin disks. The dentin surfaces were ground with \#600-grit SiC paper under running water. One surface of each disk was treated with an adhesive system according to the instructions described above. A flowable resin composite (Metafil Flo, shade A3, Sun Medical, Moriyama, Japan) was then placed between pairs of the prepared dentin disks and light-cured to make a dentin disk sandwich. After storing for 24 hours in distilled water, each prepared specimen was sectioned perpendicular to the adhesive-dentin interface with a diamond saw and

Table 1 Compositions of the experimental all-in-one adhesive systems

\begin{tabular}{|c|c|c|c|c|c|c|c|c|c|c|}
\hline \multirow{2}{*}{$\begin{array}{l}\text { Code } \\
\text { (pH value) }\end{array}$} & \multicolumn{3}{|c|}{ Functional monomer } & \multicolumn{3}{|c|}{ Base monomer } & \multicolumn{2}{|c|}{ Solvent } & \multicolumn{2}{|c|}{ Initiator } \\
\hline & MDP & 3D-SR & 4-META & Bis-GMA $^{1}$ & TEGDMA $^{2}$ & $\mathrm{HEMA}^{3}$ & Water $^{4}$ & Acetone $^{5}$ & $\mathrm{CQ}^{6}$ & $\mathrm{DMBE}^{\tau}$ \\
\hline $\begin{array}{l}\text { MDP } \\
(1.9)\end{array}$ & 12 & - & - & 14 & 10 & 12 & 10 & 40 & 1 & 1 \\
\hline $\begin{array}{l}\text { 3D-SR } \\
(2.0)\end{array}$ & - & 12 & - & 14 & 10 & 12 & 10 & 40 & 1 & 1 \\
\hline $\begin{array}{l}\text { 4-META } \\
(2.2)\end{array}$ & - & - & 12 & 14 & 10 & 12 & 10 & 40 & 1 & 1 \\
\hline
\end{tabular}

Unit: wt\%

Bis-GMA; bisphenol A-glycidyl methacrylate, CQ; camphorquinone, DMBE; p-(dimethylamino) benzoic acid ethyl ester, 3D-SR; three dimensional surface-reinforcing monomer, HEMA; 2-hydroxyethyl methacrylate, MDP; 10-methacryloxydecyl dihydrogen phosphate, 4-META; 4-methacryloxyethyl trimellitate anhydride, TEGDMA; triethyleneglycol dimethacrylate.

${ }^{1} 1215 \mathrm{H}$, Shin-Nakamura Chemical, Wakayama, Japan; ${ }^{2} 1030 Y$, Shin-Nakamura Chemical, Wakayama, Japan; ${ }^{3}$ 9101568, Kyoeisha Chemical, Osaka, Japan; ${ }^{4}$ EPN6583, Wako Pure Chemical Industries, Osaka, Japan; ${ }^{5}$ Wako Pure Chemical Industries, Osaka, Japan; ${ }^{6}$ FIL01, Tokyo Kasei Kogyo, Tokyo, Japan; ${ }^{7}$ AGN01, Tokyo Kasei Kogyo, Tokyo, Japan. 
<smiles>C=C(C)C(=O)OCCCCCO[P+](=O)(O)O</smiles><smiles>C=C(C)C(=O)OCCOC(=O)c1ccc2c(c1)C(=O)OC2=O</smiles><smiles>[Y]C(C)(C)OP(=O)(O)C([Y])(C)OCCOC(=O)C(=C)C</smiles>

MDP

\section{4-META}

Fig. 1 Chemical formulae of the functional monomers used in this study.

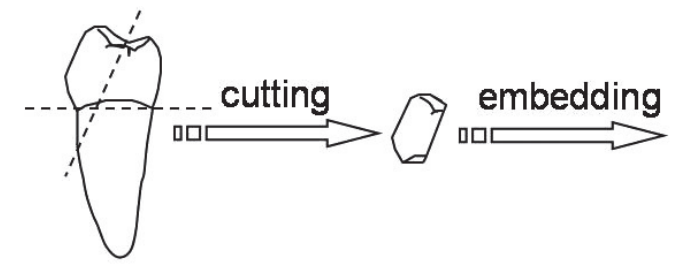

Human premolar

(For Enamel)

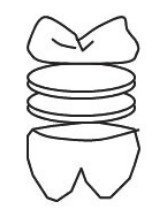

Human molar

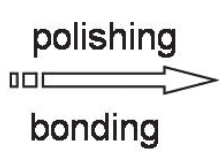

(For Dentin)

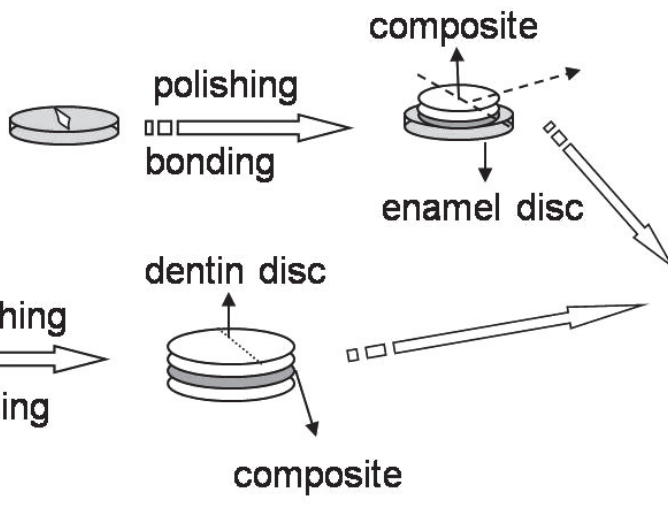

composite

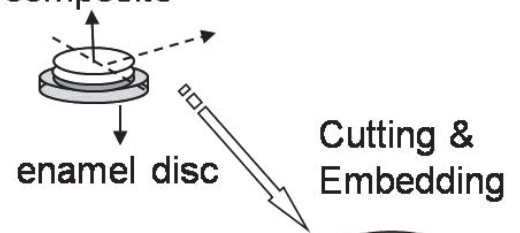

Argon-ion etching<smiles>[V]=[Tl]</smiles>

SEM

\section{Super bond $\mathrm{C} \& \mathrm{~B}$}

covering

sectioning

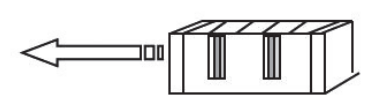

Polishing

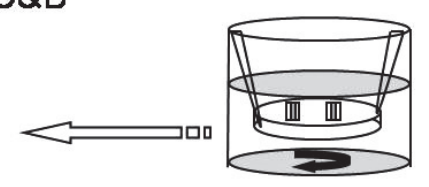

Acid-base challenge
1. demineralization solution $(\mathrm{pH} 4.5)$

2. $5 \% \mathrm{NaClO}$

Fig. 2 Illustration for specimen preparation for enamel/dentin acid-base resistant zone.

Note that the immersion periods for the acid-base challenge of the specimens were different between dentin and enamel. 
embedded with an epoxy resin (Epoxicure Resin, Buehler).

Each specimen was first stored in $100 \mathrm{~mL}$ of a buffered demineralizing solution, containing $2.2 \mathrm{mmol} / \mathrm{L}$ $\mathrm{CaCl}_{2}, 2.2 \mathrm{mmol} / \mathrm{L} \mathrm{NaH}{ }_{2} \mathrm{PO}_{4}$ and $50 \mathrm{mmol} / \mathrm{L}$ acetic acid adjusted at pH 4.5 for 90 min to create artificial secondary caries ${ }^{19)}$. The specimens were then immersed in $5 \%$ $\mathrm{NaClO}$ for $20 \mathrm{~min}$ in an attempt to remove any demineralized dentin collagen fibrils, and rinsed with running water for $30 \mathrm{~s}$. Following this, a 4-META/ MMA-TBB resin (Super Bond C\&B, Sun Medical) was applied without acid-etching of the treated surface in order to prevent wear of the adhesive during polishing, to prevent from the edge of the adhesive tearing away during specimen polishing ${ }^{7}$. After curing of the 4-META/ MMA-TBB resin, the specimens were sectioned perpendicular to the dentin-adhesive interface, and reduced to approximately $1 \mathrm{~mm}$ thickness, then polished with diamond pastes (Struers A/S, Copenhagen, Denmark) down to $0.25 \mu \mathrm{m}$. The polished surfaces were etched with an argon-ion beam (EIS-IE, Elionix, Tokyo, Japan) for 6 min to bring the hybrid layer into a sharp relief. Operating conditions for the argon-ion beam etching were an accelerating voltage of $1 \mathrm{kV}$ and an ion current density of $0.2 \mathrm{~mA} / \mathrm{cm}^{2}$, with the ion beam directed perpendicular to the polished surface. The specimens were then gold-sputter coated, and morphological changes to the adhesive-dentin interface due to acid-base challenge were observed using a SEM (JSM-5310LV, JEOL, Tokyo, Japan).

\section{Specimen preparation for enamel $A B R Z$}

Human premolars were used for this part of the study as shown in Fig. 2. Roots were removed and crowns were sectioned mesiodistally using a low speed diamond saw. In order to prepare the mid-coronal buccal enamel surfaces for bonding, crowns with buccal surfaces facing out were embedded in epoxy resin overnight and then wet-polished with \#600-grit SiC paper until flat enamel surfaces were exposed. The surfaces were examined under a stereo microscope (Nikon SMZ10, Tokyo, Japan) at $10 \times$ magnification to exclude any surface defects or decalcified areas. The enamel surfaces were treated with one of the three adhesive systems according to the directions described above. A flowable composite was then applied on the top (approximately $1 \mathrm{~mm}$ in thickness) and cured for $20 \mathrm{~s}$.

After storage in distilled water at $37^{\circ} \mathrm{C}$ for $24 \mathrm{~h}$, each specimen was sectioned perpendicular to the bonding interface into two halves and embedded in the epoxy resin overnight again. They were wet-polished with $\mathrm{SiC}$ paper from 400-grit to 1200-grit to standardize the surface, and then subjected to an acid-base challenge. For the acid challenge, each specimen was stored in 100 $\mathrm{mL}$ buffered demineralizing solution described above for $4.5 \mathrm{~h}$ to create artificial secondary caries ${ }^{19)}$. For the base challenge, the specimens were immersed in $5 \% \mathrm{NaClO}$ for $30 \mathrm{~min}$ with ultrasonic in an attempt to remove any demineralized structures or enamel proteins, and rinsed with running water for $30 \mathrm{~s}$. These acid-base challenge conditions were performed according to the previous study $^{15)}$. The SEM observation of the enamel ABRZ was then performed in the same manner described above for dentin.

\section{RESULTS}

Representative SEM images of the interfacial structures of dentin and enamel specimens after acid-base challenge were revealed in Figs. 3 and 4, respectively. An outer lesion (OL), created by mineral loss due to the acid-base challenge, was observed in all the groups. The depth of the OL ranged from 10 to $15 \mu \mathrm{m}$ in dentin specimens (Fig. 3) and from 10 to $20 \mu \mathrm{m}$ in enamel (Fig. 4).

On the SEM micrographs of the dentin specimens, similar morphologies were observed among the three adhesive groups (Figs. 3a, 3b and 3c). The adhesive layer (B) was less than $10 \mu \mathrm{m}$ under a $\times 3,500$ magnification. The dentin ABRZ was clearly observed in all the specimens. However, the ABRZ in MDP (a) and 3D-SR (b) seemed to be thicker than that in 4-META (c). Thickness of the dentin ABRZ measured at the mid
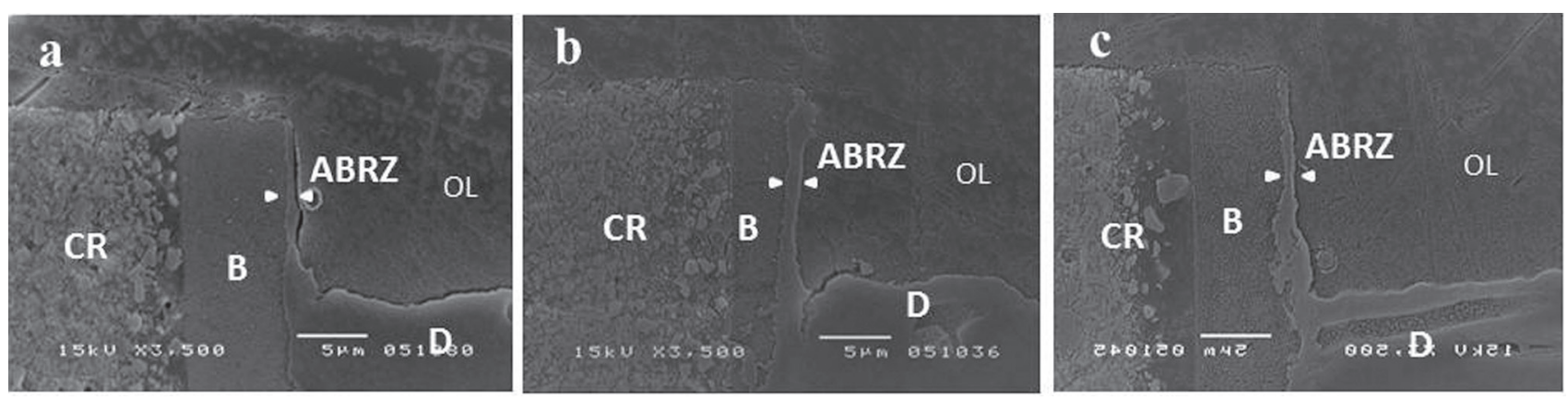

Fig. 3 SEM observations of the adhesive-dentin interface after acid-base challenge. a. MDP-dentin, b. 3D-SR-dentin, c. 4-META-dentin (magnification; $\times 3,500$ ) OL; outer lesion, CR; resin composite, B; bonding resin, ABRZ; acid-base resistant zone, D; dentin In the adhesive-dentin interface after acid-base challenge, similar ultra-morphologies were observed in three adhesive groups. A hybrid layer was hardly detected, whereas the dentin ABRZ was clearly observed in all specimens. Thickness of the ABRZ in MDP (a) and 3D-SR (b) seemed thicker than that in 4-META (c). 

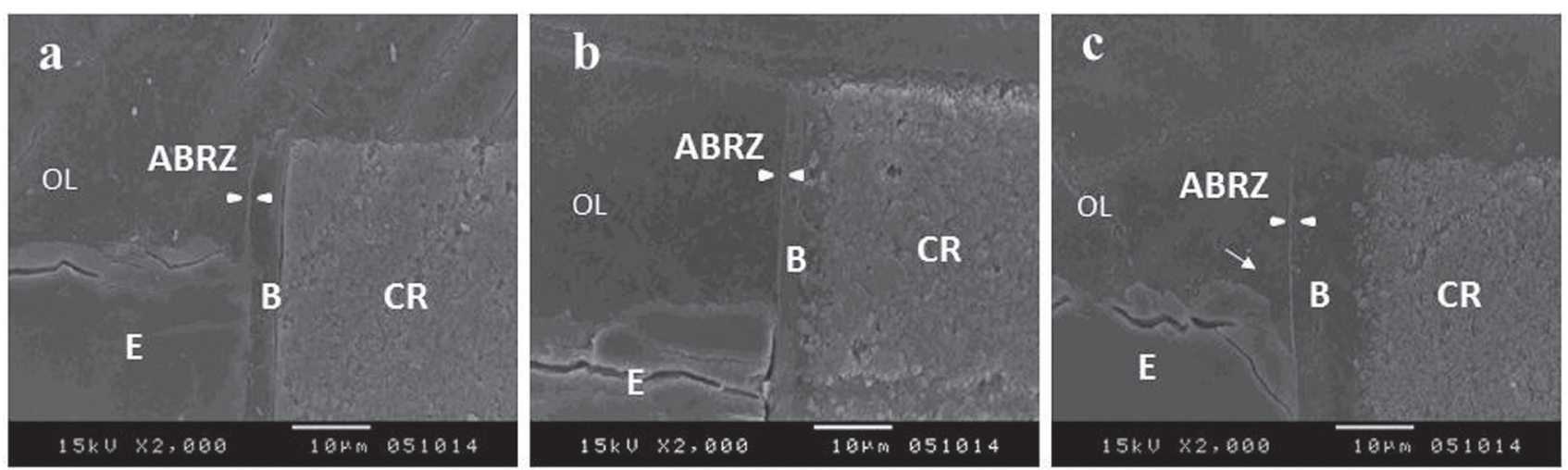

Fig. 4 SEM observations of the adhesive-enamel interface after acid-base challenge. a. MDP-enamel, b. 3D-SR-enamel, c. 4-META-enamel (magnification; ×2,000) OL; outer lesion, CR; resin composite, B; bonding resin, ABRZ; acid-base resistant zone, E; enamel. In the enamel specimens, the ABRZ was confirmed in the three adhesive groups. The enamel ABRZ of MDP (a) was thicker than those of 3D-SR (b) and 4-META (c). Funnel-shaped erosion (white arrow) at the junction of the bonding interface, enamel and OL was observed in 4-META (c).

portion of the OL was $0.8-1.0 \mu \mathrm{m}$ in MDP, $0.7-1.0 \mu \mathrm{m}$ in 3D-SR, and 0.6-0.7 $\mu \mathrm{m}$ in 4-META, respectively.

Also in the enamel specimens (Fig. 4), the presence of an ABRZ was confirmed at the adhesive interface. In comparison with the dentin ABRZ, the enamel ABRZ was very thin, measuring less than $0.5 \mu \mathrm{m}$ in each adhesive. In addition, the ABRZ formed on enamel with MDP (a) appeared to be thicker than those of 3D-SR (b) and 4-META (c) among the three adhesive systems. A funnel-shaped erosion (white arrow) at the junction of the bonding interface between enamel and OL was observed in the 4-META group (c).

\section{DISCUSSION}

Sample preparation for SEM observation of dentin ABRZ was established by Inoue et $a l .{ }^{7}{ }^{7}$ and successfully applied in many studies afterwards $s^{8-13,20)}$. However, considering the structural differences between dentin and enamel, it was necessary to seek optimal conditions for the acid-base challenge in order to observe enamel ABRZ. According to the previous study ${ }^{15}$, in order to create outer lesions with depths comparable to those created with dentin, the enamel specimens were subjected to the same demineralizing solution for $4.5 \mathrm{~h}$, which was three times longer than the challenge time for dentin. On the other hand the variety in depth of the OL created seemed to be wider in enamel than in dentin, which was probably due to the longer immersion period of enamel specimens in a demineralization solution for acid challenge. For the base challenge, $5 \% \mathrm{NaOCl}$ solution was applied for 30 min with ultrasonic vibration to completely remove the demineralized enamel proteins.

The ABRZ was validated at the bonding interface both in the dentin and enamel specimens. However, morphological differences in ABRZ were observed for each adhesive system in both enamel and dentin. In the dentin specimens, morphological features of the interface were similar among the three adhesive systems. However, the dentin ABRZs of MDP and 3D-SR seemed to be slightly thicker than that of 4-META (Fig. 3), which was probably due to different aggressiveness of each adhesive. The acidity of a self-etch adhesives depends on compositional factors. In this regard, if the experimental adhesives were sorted according to their $\mathrm{pH}$ values, the asending order is $\mathrm{MDP}<3 \mathrm{D}-\mathrm{SR}<4$-META, respectively. The acid dissociation constant (pKa) of the acidic monomer in each adhesive is also generally considered to be one of the major parameters in determining the potential of the adhesive to decalcify mineralized tissues ${ }^{21}$. The $\mathrm{pKa}$ values of the functional monomers in water were as follows: MDP; pKa1 $=2.8$, pKa2 $=6.9$ (technical report from Kuraray Medical), and 4-MET (hydrolyzed 4-META); pKa1=2.7, pKa2=4.7 (technical report from Sun Medical). However, The $\mathrm{pKa}$ value of 3D-SR was unknown. In addition to $\mathrm{pKa}$, the potential of each acidic monomer to dissolve HAp is also negatively influenced by their molecular weight. It should be also noted that while etching is required to promote penetration of monomers and adhesion to HAp, the etching capacity does not fully explain the mechanisms of adhesion to HAp, as the mechanism also depends on the dissolution rate of the calcium salt formed with each acidic monomer in the acid solution ${ }^{21}$.

The formation of an enamel ABRZ was also confirmed along the adhesive-enamel interface in all the groups in the study. The enamel ABRZ was thinner than the dentin ABRZ for each group. A funnel-shaped erosion area was observed specifically in the 4-META adhesive. Inoue $e t a l .{ }^{11)}$ reported that the dentin surface adjacent to the ABRZ was more eroded than other areas of the dentin surface in a 4-META containing all-in-one adhesive (AQ Bond Plus, Sun Medical). They suggested that nanospaces caused by incomplete monomer penetration into the demineralized dentin should be present beneath the dentin ABRZ. Li et al. ${ }^{15)}$ reported 
that a funnel-shaped erosion area was noted in the bonded specimen in enamel when Phenyl-P was incorporated in both the primer and the bonding agents of an experimental two-step self-etching adhesive system, while the enamel ABRZ was clearly detected when MDP was included either in the primer or in the bonding agent. These studies clearly demonstrated that functional monomers in the adhesive systems strongly influenced the morphology of the enamel/dentin ABRZ.

It was speculated that the differences observed among enamel ABRZs in the current study reflects the chemical characteristics of each acidic monomer with regard to the dissolution rate of the calcium salt formed, as mentioned above for dentin. Yoshihara et al. ${ }^{22}$ elucidated the mechanisms of interfacial interaction of a functional monomer with HAp particles in a theoretical model. According to their X-ray diffraction and solid-state nuclear magnetic resonance studies, each of the three functional monomers; MDP, 4-MET and Phenyl-P interacted with HAp differently. Ionic bond of MDP with calcium appeared to be hydrolytically stable, as compared with 4-MET and Phenyl-P ${ }^{3}$. It was capable of forming strong ionic bonds with calcium due to superficial dissolution of HAp induced by the MDP adsorption and subsequent deposition of MDP-calcium salt with a lower solubility. In contrast, the chemical bonding capacity of 4-MET was limited. The resulting Ca-salts are susceptible to dissolution during the acid-base challenge.

From the current results, it was inferred that the soluble stability of 3D-SR was probably close to that of MDP. As the chemical structure of 3D-SR illustrated in Fig. 1 suggested the phosphate monomer could partially self-organize within adhesive, and form multifunctional monomer structures with several phosphate groups and polymerizing groups. Those several phosphate groups in the 3D-SR monomer are capable to interact with calcium at multiple sites and form ionic bonds. It is expected that by air-blowing the adhesive applied on the tooth substrate, the 3D-SR monomers are concentrated at the resin-tooth interface and crosslink with calcium. It was suggested that a 3D-SR-containing all-in-one adhesive, Bond Force (Tokuyama Dental), might have improved the incomplete polymerization and lower degree of conversion of the resin monomers that occur in many one-bottle self-etch adhesives ${ }^{23)}$. Tsujimoto et al. ${ }^{13)}$ reported that the thickness value of the dentin ABRZ created by Bond Force was approximately $0.6 \mu \mathrm{m}$ at the middle portion. Moreover, the dentin ABRZ showed a slope-like increase in the thickness from the top to the bottom of the outer lesion, probably due to its fluoride release. Such slope formation was not observed in the current study, since the experimental adhesive systems were fluoride-free. Adding up the findings of the current study and those of the previous ones ${ }^{8,9,12,13)}$, it was understood that the dentin ABRZ formation was influenced by not only the functional monomers, but also fluoride-release from the adhesive systems. Accordingly, the hypothesis of this study was accepted. Selection of the functional monomers in the adhesives may also influence the formation of both the dentin ABRZ and the enamel ABRZ. However, further study should be carried out to elucidate the effect of ingredients in adhesive systems on the ABRZ formation in both enamel and dentin.

The dentin ABRZ has a dentin-like structure with hydroxyapatite crystallites, which is more acid-base resistant than normal dentin ${ }^{24)}$. Therefore, the new terminology "super dentin" was proposed in the dentin $\mathrm{ABRZ}^{10,14)}$. On the other hand, the micromechanical adhesion, achieved by the diffusion and polymerization of resin monomers into the pretreated enamel, is assumed to be the principal bonding mechanism of dental adhesives to enamel ${ }^{6}$. Meanwhile, the ABRZ was observed in enamel, and may be similar in nature to that reported for dentin. Compared to dentin structure, enamel has a higher mineral content with a matrix structure different from dentin collagen network, with lower depth of demineralization and HAp crystals distributed all over the demineralized layer. Therefore, the dentin ABRZ forms under the hybrid layer; while the enamel ABRZ was created along the interface between adhesive and enamel. Since the enamel ABRZ demonstrated more acid-base resistant than intact enamel, the enamel ABRZ could be named "super enamel".

Despite adhesive-dental hard tissue interfaces having been thoroughly studied ultra-morphologically, the complex molecular interactions at the interface have hardly been investigated and are far from understood ${ }^{25)}$. The methodology to observe the ABRZ has been established to examine the ultra-structures of the secondary caries formation at the adhesive-dentin interface $^{7}$. However, this may be helpful to bridge the knowledge among the clinical aspect and bonding mechanisms. Interchanging a single functional monomer in an experimental adhesive system is an interesting model for investigating bonding mechanisms and considering chemical interactions with dental substrates. Further study should be carried out to elucidate the relationship between the ABRZ patterns of the different adhesive systems and the long-term enamel/dentin bond durability. The ABRZ pattern obtained from each adhesive system may become a good indicator to predict bonding durability to tooth substrates.

\section{CONCLUSIONS}

1. The ABRZ formation was confirmed at both enamel and dentin bonding interface treated with the three adhesive systems.

2. Morphology of the ABRZ was influenced by the functional monomers contained in the adhesive systems.

\section{ACKNOWLEDGMENT}

The authors thank Tokuyama Dental Co. for supplying the experimental materials. This work was supported by the grant from the Global Center of Excellence (GCOE) Program, International Research Center for Molecular 
Science in Tooth and Bone Diseases at Tokyo Medical and Dental University and a Grant-in Aid for the Japan Society for the Promotion of Science (JSPS No. 22592116).

\section{REFERENCES}

1) Van Landuyt KL, Snauwaert J, De Munck J, Peumans M, Yoshida Y, Poitevin A, Coutinho E, Suzuki K, Lambrechts P, Van Meerbeek B. Systematic review of the chemical composition of contemporary dental adhesives. Biomaterials 2007; 28: 3757-3785.

2) Van Landuyt KL, Yoshida Y, Hirata I, Snauwaert J, De Munck J, Okazaki M, Suzuki K, Lambrechts P, Van Meerbeek $B$. Influence of the chemical structure of functional monomers on their adhesive performance. J Dent Res 2008; 87: 757-761.

3) Yoshida Y, Nagakane K, Fukuda R, Nakayama Y, Okazaki M, Shintani H, Inoue S, Tagawa Y, Suzuki K, De Munck J, Van Meerbeek B. Comparative study on adhesive performance of functional monomers. J Dent Res 2004; 83: 454-458.

4) Inoue S, Koshiro K, Yoshida Y, De Munck J, Nagakane K, Suzuki K, Sano H, Van Meerbeek B. Hydrolytic stability of self-etch adhesives bonded to dentin. J Dent Res 2005; 84: 1160-1164.

5) Tsuchiya S, Nikaido T, Sonoda H, Foxton RM, Tagami J. Ultrastructure of the dentin-adhesive interface after acid-base challenge. J Adhes Dent 2004; 6: 183-190.

6) Nakabayashi N, Kojima K, Masuhara E. The promotion of adhesion by the infiltration of monomers into tooth substrates. J Biomed Mater Res 1982; 16; 265-273.

7) Inoue G, Tsuchiya S, Nikaido T, Foxton RM, Tagami J. Morphological and mechanical characterization of the acid-base resistant zone at the adhesive-dentin interface of intact and caries-affected dentin. Oper Dent 2006; 31: 466-472.

8) Shinohara MS, Yamauti M, Inoue G, Nikaido T, Tagami J, Giannini M, de Goes MF. Evaluation of antibacterial and fluoride-releasing adhesive system on dentin-microtensile bond strength and acid-base challenge. Dent Mater J 2006; 25: 545-552

9) Waidyasekera K, Nikaido T, Weerasinghe DDS, Ichinose S, Tagami J. Reinforcement of dentin in self-etch adhesive technology: a new concept. J Dent 2009; 37: 604-609.

10) Nikaido T, Weerasinghe DDS, Waidyasekera K, Inoue G, Foxton RM, Tagami J. Assessment of the nanostructure of acid-base resistant zone by the application of all-in-one adhesive systems: Super dentin formation. Biomed Mater Eng 2009; 19: 163-171.

11) Inoue G, Nikaido T, Foxton RM, Tagami J. The acid-base resistant zone in three dentin bonding systems. Dent Mater J 2009; 28: 717-721.

12) IIda $Y$, Nikaido $T$, Kitayama $S$, Takagaki $T$, Inoue G, Ikeda M, Foxton RM, Tagami J. Evaluation of dentin bonding performance and acid-base resistance of the interface of two-step self-etching adhesive systems. Dent Mater J 2009; 28: 493-500.

13) Tsujimoto M, Nikaido T, Inoue G, Sadr A, Tagami J. Ultrastructural observations of the acid-base resistant zone of all-in-one adhesives using three different acid-base challenges. Dent Mater J 2010; 29: 655-660.

14) Nikaido T, Inoue G, Takagaki T, Waidyasekera $K$, Iida $Y$, Shinohara MS, Sadr A, Tagami J. New strategy to create "Super Dentin" using adhesive technology: Reinforcement of adhesive-dentin interface and protection of tooth structures. Jpn Dent Sci Rev 2011; 47: 31-42.

15) Li N, Nikaido T, Takagaki T, Sadr A, Makishi P, Chen J, Tagami J. The role of functional monomers in bonding to enamel: acid-base resistant zone and bonding performance. J Dent 2010; 38: 722-730.

16) Takeyama M, Kashibuchi S, Nakabayashi N, Masuhara E. Studies on dental self-curing resins (17). Adhesion of PMMA with bovine enamel or dental alloys. Shika Rikogaku Zassi 1978; 19: 179-185.

17) Omura I, Yamauchi J, Nagase Y, Uemura F, inventors; Kuraray Co., Ltd., assignee. Adhesive composition. US patent 4539382, 1985; Sep 3.

18) Dodomi A, Matsushige K, Suzuki K, inventors; Tokuyama Dental Co. Ltd. assignee. Adhesive composition. JP 2009-29743 A, 2009; Feb 12.

19) Wefel JS, Jensen ME, Triolo PT, Faller RV, Hogan MM, Bowman WD. Re/remineralization from sodium fluoride dentifrices. Am J Dent 1995; 8: 217-220.

20) Takagaki T, Nikaido T, Tsuchiya S, Ikeda M, Foxton RM, Tagami J. Effect of hybridization on bond strength and adhesive interface after acid-base challenge using 4-META/ MMA-TBB resin. Dent Mater J 2009; 28: 185-193.

21) Yoshida Y, Van Meerbeek B, Nakayama Y, Yoshioka M, Snauwaert J, Abe Y, Lambrechts P, Vanherle G, Okazaki M. Adhesion to and decalcification of hydroxyapatite by carboxylic acids. J Dent Res 2001; 80: 1565-1569.

22) Yoshihara K, Yoshida Y, Nagaoka N, Fukegawa D, Hayakawa S, Mine A, Nakamura M, Minagi S, Osaka A, Suzuki K, Van Meerbeek B. Nano-controlled molecular interaction at adhesive interfaces for hard tissue reconstruction. Acta Biomater 2010; 6: 3573-3582.

23) Hosoya Y, Tay FR, Ono T, Miyazaki M. Hardness, elasticity and ultrastructure of primary tooth dentin bonded with a self-reinforcing one-step self-etch adhesive. J Dent 2010; 38: 214-221.

24) Waidyasekera PGK, Nikaido T, Weerasinghe DDS, Wettasinghe KA, Tagami J. Caries susceptibility of human fluorosed enamel and dentine. J Dent 2007; 35: 343-349.

25) Eisenburger M. Degree of mineral loss in softened human enamel after acid erosion measured by chemical analysis. $J$ Dent 2009; 37: 491-494. 